\title{
Preliminary observations of chondral abrasion in a canine model
}

\author{
Roy D Altman, Jonathan Kates, Lawrence E Chun, David D Dean, David Eyre
}

Arthritis Section, Miami Veterans Administration Medical Center, Miami, Florida, USA R D Altman D D Dean

Department of Orthopedics and Rehabilitation, University of Miami School of Medicine, Miami, Florida, USA J Kates

Department of Orthopaedics, University of Washington, Seattle, Washington, USA

L E Chun

D R Eyre

Department of

Medicine and

Biochemistry-

Molecular Biology,

University of Miami

School of Medicine, Miami, Florida, USA

D D Dean

Department of Medicine, University of Miami School of Medicine, Miami, Florida, USA R D Altman

Correspondence to: Dr Roy D Altman, Arthritis Section (111), Miami Veterans

Administration Medical

Administration Medical Room D-1009, Miami, FL 33125, USA.

Accepted for publication 27 April 1992

\begin{abstract}
Articular cartilage repair was followed for one year in skeletally mature dogs after destabilisation by anterior cruciate ligament transection of the stifle joint (CT), abrasion of the inferior medial condyle (ABR) to bleeding bone, or anterior cruciate transection followed by chondral abrasion (CT/ABR). ABR animals formed repair cartilage at the abrasion site (ABR and CT/ABR) at six months as determined by arthroscopy and at necropsy. CT and CT/ABR animals had an additional cartilage ulcer on the superior aspect of the medial condyle. The abraded site extended in CT/ABR condyles. Repair cartilage (ABR and CT/ABR) contained reduced amounts of proteoglycan as seen by histological loss of safranin $\mathbf{O}$ staining and reduced uronic acid content. Fibrocartilage was suggested by histological appearance, hypocellularity, and a higher hydroxyproline content. In contrast with $A B R$ animals, the repair cartilage in the CT/ABR animals contained near normal amounts of hydroxyproline. Collagen profiles of abrasion site repair cartilage in ABR animals had more types $I$ and $V$ collagens, similar amounts of type VI collagen, and decreased amounts of types II, IX, and XI collagens than $\mathrm{CT} / \mathrm{ABR}$ animals. The results of this study are consistent with abrasion chondroplasty leading to a repair cartilage. Despite extended ulcers, repair cartilage from the destabilised joint (CT/ABR) animals was more hyaline-like in its hydroxyproline content and collagen composition than repair cartilage from the stable joint (ABR animals). In these models additional measures appear to be needed as the defects induced by abrasion chondroplasty did not form a functional hyaline cartilage.
\end{abstract}

(Ann Rheum Dis 1992; 51: 1056-1062)

Osteoarthritis is a common joint disease characterised by loss of cartilage and exposure of underlying subchondral bone. Current treatment does not address the maintenance of cartilage or its repair but is directed at joint symptoms and seeks to reduce pain and maintain joint function.

Previous studies provide evidence that articular cartilage in mature animals and humans can repair if the subchondral plate is penetrated. ${ }^{1-11}$ Abrasion chondroplasty is based on this concept and involves the surgical removal of superficial areas of 'dead' bone from the arthritic joint until bleeding cortical bone is exposed. ${ }^{12-19}$ The exposure of subchondral cancellous bone in this procedure seems to stimulate new cartilage formation ${ }^{1820}$ and suggests that it may provide access to pleuripotential cells that can form new cartilage. ${ }^{21}$ Abrasion chondroplasty of the knee has been developed for use in humans by Johnson. ${ }^{17}$

This trial was designed to describe the character and nature of the repair cartilage formed following abrasion chondroplasty in the canine knee (stifle) joint. To simulate a clinical setting the joint was destabilised before abrasion in a subset of dogs. Tissue repair was followed up for one year.

\section{Materials and methods}

EXPERIMENTAL ANIMALS

Nine mongrel dogs weighing 18-25 kg were screened for signs of illness and skeletal maturity by joint radiography. Although adult their exact ages were unknown. All operations were performed under intravenous pentobarbital anaesthesia (30 mg/kg body weight) and endotracheal intubation. Following the operations the animals were placed in their cages and detubated when spontaneous breathing returned. The dogs were ambulant within 12 hours of recovery and in three days were allowed free exercise in open kennels for a minimum of one hour daily. For three days after the operation all animals were examined for haemarthrosis and infection. Animals were fed Hi-Pro Dog Chow (Purina Laboratories) and had free access to water, and under these conditions changed no more than $10 \%$ body weight during the one year follow up.

The dogs were randomly divided into three groups.

Induction of osteoarthritis by cruciate ligament transection: $C T$ group

The anterior cruciate ligament of the right knee (stifle joint) was transected by a $1 \mathrm{~cm}$ percutaneous medial parapatellofemoral stab incision into the intercondylar groove with a No 11 Bard-Parker surgical blade as described by Pond and Nuki. ${ }^{22}$ The wound was sutured after active bleeding had stopped. Excess blood was irrigated from the joint with saline. Before recovery from anaesthesia the completeness of the transection was verified by an anteriorposterior 'Drawer sign'.

Abrasion chondroplasty of normal articular cartilage: $A B R$ group

Diagnostic arthroscopy was performed on the 
right knee through medial and lateral inferoanterior portals to verify the presence of normal synovium, ligaments, and articular surface. The arthroscopy was performed using a $5.5 \mathrm{~mm} 15^{\circ}$ off angle arthroscope (Storz, Culver City, CA, USA) with suprapatellar sac inflow of saline. No tourniquet was applied and the procedure was videotaped. Abrasion chondroplasty was performed to the anteroinferior medial surface of the medial condyle of the right knee using a Dyonics abrasion instrument system burr (Dyonics, Woburn, MA, USA). A $5 \mathrm{~mm}^{2}$ ulcer was produced in the cartilage to expose underlying bone (Outerbridge stage IV). ${ }^{23}$ Surface vessels were exposed by trimming $\leqslant 1 \mathrm{~mm}$ of surface bone and no red bone was exposed. Blood was observed to stream from scattered areas of the abraded surface.

\section{Abrasion chondroplasty of $C T$ induced cartilage lesions: CT/ABR group}

The right knee was destabilised by cruciate transection as described earlier. After two weeks of activity the abrasion procedure was performed as described in the preceding section. Although the animal does not have a mature osteoarthritic lesion at two weeks it allows examination of the combination of joint destabilisation with abrasion chondroplasty.

After six months' activity videotaped diagnostic arthroscopy was performed on all animals. The entire knee was examined with particular attention to the abraded areas and weight bearing surfaces.

The study was terminated at 12 months. Knee radiographs were obtained at the time of pentobarbital induced euthanasia. The contralateral (unoperated) left knee was used as a normal control. Earlier studies had shown the unoperated canine knee to be essentially normal based on gross pathology, histology and biochemistry. ${ }^{24}$

\section{GROSS AND MICROSCOPIC ANATOMY}

Gross anatomy of the articular surfaces and joint structures were noted at six months by arthroscopy and at 12 months at necropsy. Gross synovial inflammation was recorded as absent $(0)$, mild $(+)$, moderate $(++)$, or marked $(+++)$. Cartilage surfaces were characterised as normal (N) if white, smooth, and elastic. Abnormal cartilage surfaces were graded according to the system of Outerbridge: ${ }^{23}$ stage I, soft, discoloured, superficial fibrillation; stage II, fragmentation $<1.3 \mathrm{~cm}^{2}$; stage III, fragmentation $>1.3 \mathrm{~cm}^{2}$; stage IV, erosion to subchondral bone. Cruciate ligaments were noted as present $(\mathrm{P})$ or absent (A). Meniscal changes were graded as normal $(\mathrm{N})$, containing horizontal cleavage tears (1), vertical tears (2), or disruption of the anterior attachment (3). Osteophytes were noted as present $(\mathrm{P})$ or absent (A) when the dogs were killed.

Small sections of cartilage from the weight bearing area of each compartment were fixed in $30 \%$ formaldehyde and then embedded in paraffin for histology. Alternate sections were stained with safranin $O$ or haematoxylin-eosin and graded for degree of osteoarthritis according to the scale of Mankin et al $2>$ by a single blinded investigator. The Mankin scale ranges from 0 (normal cartilage) to 14 (severe disease) and is derived from four separate subscores for cellularity, safranin $\mathbf{O}$ staining, cartilage structure (fissures), and tidemark integrity. Abraded areas and areas of surface irregularity were collected and graded separately. All remaining articular cartilage was rinsed in physiological saline and stored in saline at $-80^{\circ} \mathrm{C}$ until assayed.

Synovial specimens were similarly fixed in $30 \%$ formaldehyde, embedded in paraffin, sectioned, and stained with haematoxylin-eosin. Microscopic synovitis was graded as absent (0), mild $(+)$, moderate $(++)$, or marked $(+++)$.

\section{DETERMINATION OF URONIC ACID AND} HYDROXYPROLINE

Cartilage samples (12-15 mg wet weight immediately after killing) were digested according to the method of Oegema et al ${ }^{26}$ in $300 \mu \mathrm{l} 0.1 \mathrm{M}$ sodium phosphate buffer, $\mathrm{pH} 6.5$, containing $0.01 \mathrm{M}$ EDTA and $150 \mu \mathrm{g}$ proteinase K (Sigma; type XI from Tritirachium album). After 18 hours at $56^{\circ} \mathrm{C}$ the digests were cooled to room temperature and assayed for their uronic acid and hydroxyproline contents. For uronic acid assays, $10 \mu \mathrm{l}$ of digest was made up to a final volume of $50 \mu \mathrm{l}$ with distilled water. The method of Bitter and Muir ${ }^{27}$ was modified only with reduction of reagent volumes to compensate for the relatively small volume of sample.

The hydroxyproline content of the digest was determined by the method of Morales $e t$ al ${ }^{28}$ followed by the spectrophotometric assay of Woessner. ${ }^{29}$ After treatment with proteinase $\mathrm{K}$, $100 \mu \mathrm{l}$ of digest was transferred to a Microflex tube (Kontes) and an equal volume of $12 \mathrm{M} \mathrm{HCl}$ was added. The tubes were sealed with PTFE lined caps and incubated at $110^{\circ} \mathrm{C}$ for 18 hours. The samples were then dried on a steam bath in a gentle stream of air. The dried samples were resuspended in $200 \mu \mathrm{l}$ of a 1:4 dilution of the original citric acid buffer and $5 \mu \mathrm{l}$ of $0.02 \%$ methyl red was added. The $\mathrm{pH}$ was adjusted to 6.0 and the volume to $250 \mu$ l. The spectrophotometric assay was then performed reducing the volume of each reagent to give a final volume of $1.25 \mathrm{ml}$. After colour development the samples were extracted twice with $900 \mu l$ of toluene to remove interfering substances and the absorbance was measured at $557 \mathrm{~nm}$.

\section{COLLAGEN ISOLATION AND CHARACTERISATION} Canine articular cartilage was dissected from the femoral condyles and sites of abrasion or ulceration immediately after euthanasia and frozen in saline at $-80^{\circ} \mathrm{C}$ until analysed.

The cartilage was first extracted by digestion with chondroitinase $A B C(0.125 \mathrm{U} / \mathrm{ml}$ at $40 \mathrm{mg}$ wet weight of tissue/ml for 24 hours at $37^{\circ} \mathrm{C}$ ) and then $4 \mathrm{M}$ guanidine $\mathrm{HCl}, 0.05 \mathrm{M}$ TRIS, pH $7 \cdot 5$, for 24 hours at $4^{\circ} \mathrm{C}$ to remove proteoglycans. ${ }^{30}$ Protease inhibitors $(2 \mathrm{mM}$ phenylmethylsulphonyl fluoride, $2 \mathrm{mM}$ EDTA, $10 \mathrm{mM} \mathrm{N}$-ethylmaleimide, and $5 \mathrm{mM}$ benzamidine $\mathrm{HCl}$ ) were included in the two extractants. 
The residual tissue was then extensively washed in saline at $4^{\circ} \mathrm{C}$ and digested with pepsin (1:20 wet weight) in $3 \%$ acetic acid for 24 hours at $4^{\circ} \mathrm{C}$. Insoluble material was removed by centrifugation.

Different collagen fractions were then precipitated from the acid solution at $0.8,1.2$, and $2.0 \mathrm{M} \mathrm{NaCl}$. The $1.2 \mathrm{M}$ fraction, enriched in type XI/V collagen chains, was dissolved in $3 \%$ acetic acid and reprecipitated at $1.2 \mathrm{M} \mathrm{NaCl}$ after removing residual type II collagen at $0.9 \mathrm{M}$ $\mathrm{NaCl}$. Type IX collagen was recovered in the $2.0 \mathrm{M}$ fraction and further purified by reprecipitation with $2.0 \mathrm{M} \mathrm{NaCl}$ from $3 \%$ acetic acid after again removing material precipitating at $1 \cdot 2 \mathrm{M} \mathrm{NaCl}$.

\section{GEL ELECTROPHORESIS}

Samples were run on sodium dodecyl sulphate$7 \cdot 5 \%$ polyacrylamide slab gels according to the method of Laemmli ${ }^{31}$ under either reducing or non-reducing conditions. The various collagens were identified by comparison with standards of the proteins prepared from bovine tissues.

\section{SAMPLING AND STATISTICAL ANALYSIS}

All samples were processed without knowledge of their origin. When appropriate, results of specimens were averaged and reported as the mean (SD). Comparisons were made by Student's $t$ test. If significance was achieved the results were compared by rank sum analysis. ${ }^{32}$

\section{Results}

\section{GROSS ANATOMY}

Following the operation all animals were active with a noticeable limp from the right hindlimb. No limb atrophy was observed during follow up. Table 1 shows the findings from arthroscopy at six months and gross pathology at one year.

Arthroscopically, at six months, the abraded area of $A B R$ and $C T / A B R$ joints was covered by a soft, fluffy, white repair cartilage (fig 1). The abraded areas had enlarged in the CT/ABR joints, whereas the remaining articular cartilage appeared normal. In CT and CT/ABR joints a separate ulcer was also found on the upper medial condyle and slight synovitis. Complete

Table 1 Gross anatomical observations of operated stifle joints at six and 12 months. Two values given for each item are six months/12 months; six month values are from arthroscopy; 12 month values at necropsy

\begin{tabular}{|c|c|c|c|}
\hline & $C I$ & $A B R$ & $C ' I / A B R$ \\
\hline $\begin{array}{l}\text { Synovial inflammation } \\
\text { Patellar cartilage*" } \\
\text { Medial condyle"* } \\
\text { Abraded area* } \\
\text { Medial meniscust } \\
\text { Medial tibial plateau } \\
\text { Anterior cruciate } \\
\text { Posterior cruciate } \\
\text { Lateral condyle" } \\
\text { Lateral meniscusł } \\
\text { Lateral tibial plateau" } \\
\text { Osteophytes. }\end{array}$ & $\begin{array}{l}+/+ \\
\text { N/N } \\
\text { N/I } \\
\text { ND/ND } \\
1 / 1,3 \\
\text { N/N } \\
\text { A/A } \\
\text { P/P } \\
\text { N/I } \\
\text { N/N } \\
\text { N/N } \\
\text { ND/P }\end{array}$ & $\begin{array}{l}O / \mathbf{O} \\
\text { N/N } \\
\text { N/N } \\
\text { II/II } \\
\text { N/N } \\
\text { N/N } \\
\text { P/P } \\
\text { P/P } \\
\text { N/N } \\
\text { N/N } \\
\text { N/N } \\
\text { ND/A }\end{array}$ & $\begin{array}{l}+/+ \\
\text { N/N } \\
\text { I/I } \\
\text { II/II } \\
1 / 1 \\
\text { N/N } \\
\text { A/A } \\
\text { P/P } \\
\text { N/I } \\
\text { N/N } \\
\text { N/N } \\
\text { ND/P }\end{array}$ \\
\hline
\end{tabular}

Abbreviations: $\mathrm{CT}=$ transection of anterior cruciate ligament; $\mathrm{ABR}=$ abrasion chondroplasty; $\mathrm{N}=$ normal, $\mathrm{I}=$ mild abnormalities, $\mathrm{P}=$ present, $\mathrm{A}$ or $\mathrm{O}=\mathrm{absent}, \mathrm{ND}=$ not observed. See under Methods for complete grading system.

"Outerbridge stage I-IV.

$+\mathrm{N}=$ normal, $1=$ horizontal cleavage tears, $3=$ disruption anterior meniscal attachment. severance of the anterior cruciate ligament was verified in CT and CT/ABR joints.

After one year the gross pathology at necropsy was similar to that seen by arthroscopy at six months. There was mild synovitis with a thickened synovial capsule in CT and CT/ABR joints. Surgically abraded regions were covered by a soft, fluffy, thin 'repair' cartilage with small areas of exposed subchondral bone. A linear extension of the contact area ulcer along the condyle was noted in CT/ABR joints (fig 2). Cartilage in the joints of CT and CT/ABR animals was slightly yellowed and softened over the condyles, particularly on the medial condyle. In ABR and CT/ABR joints the cartilage surrounding the abrasion site was dulled and pitted with overt ulceration on the upper medial condyle. The CT and CT/ABR joints showed horizontal cleavage tears of the medial menisci, half with disruption of the anteriolateral medial meniscal attachment. The CT and CT/ABR joints had osteophytes along the outer margins of the intercondylar groove and the anterior condyles (fig 3).

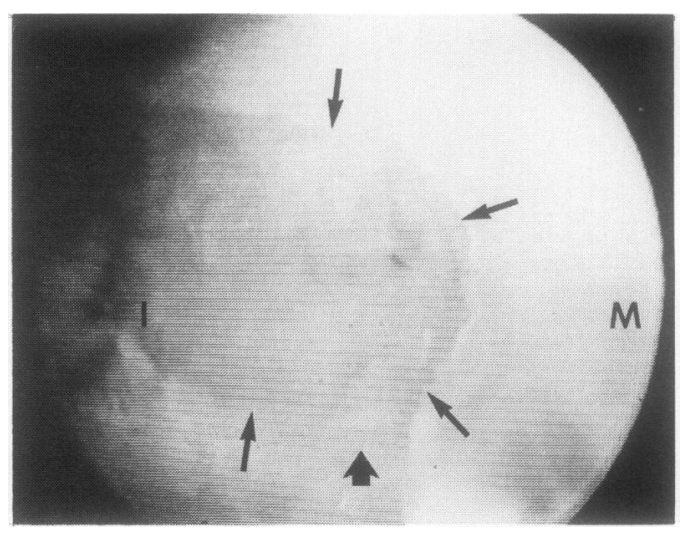

Figure 1 Videotaped arthroscopy of medial condyle in a $C T / A B R$ animal six months after abrasion chondroplasty. $A$ persistent margin (arrows) around the abraded area contains soft, fluffy repair cartilage. Extension of the ulcerated cartilage can also be seen (broad arrow). $M=$ medial condyle; $I=$ intercondylar area.

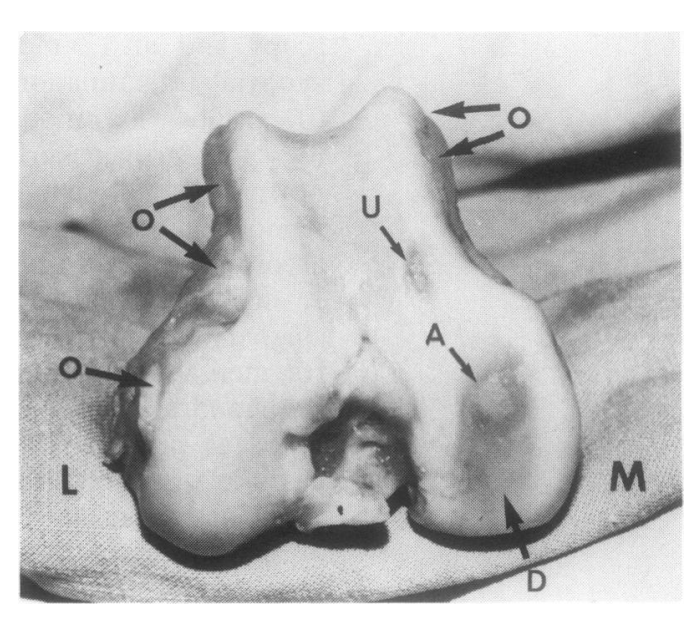

Figure 2 Distal femur at necropsy from an animal that one year previously received cruciate transection followed in two weeks by abrasion chondroplasty. The abraded site shows a soft, fluffy repair cartilage $(A)$ with distal extension of the ulcer $(D)$. The presence of a more proximal ulcer on the upper medial condyle $(U)$ can be seen in addition to marginal osteophytes $(O)$. Unaffected condylar surfaces also show superficial pitting. $M=$ medial condyle; $L=$ lateral condyle. 
Cartilage from unoperated joints was white and glistening. These animals showed no evidence of synovitis, had normal menisci, and intact cruciate ligaments.

Radiographs were obtained at one year. The

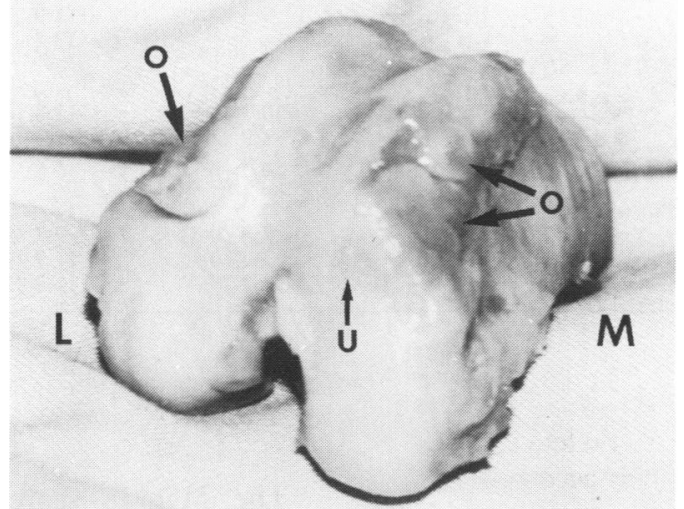

Figure 3 Distal femur at necropsy from an animal that one year previously received cruciate transection. There is an ulcer on the upper medial condyle $(U)$ in addition to marginal osteophytes $(O)$. Unaffected condylar surfaces also show superficial pitting. $M=$ medial condyle; $L=$ lateral condyle.
ABR joints showed no abnormalities. Radiographs of CT and CT/ABR joints showed normal radiographic joint space with small osteophytes on the medial, lateral, and posterior tibial plateaus and anterosuperiorly in the condyles. There were no subchondral abnormalities. Kellgren and Lawrence grade II osteoarthritis was noted based on the presence of definite osteophytes on the radiograph. ${ }^{33}$ The radiographic osteophytes were verified at necropsy.

\section{MICROSCOPIC ANATOMY}

The histological appearance of contralateral, unoperated control cartilage was normal (fig 4A). All abraded sites were partially covered by a thin cartilage that was mostly hypercellular but hypocellular in the central third (fig 4B). No site was fully repaired. Qualitatively the repair tissue at one year 'appeared' thinner than that found at six months. Cartilage surrounding the abraded and ulcerated areas of the medial condyle showed slight cell cloning and partial loss of safranin $\mathrm{O}$ staining. The $\mathrm{CT}$ and CT/ABR joints had upper medial condylar ulcers that were mostly superficial and contained chondrocyte clones (fig 4C).

\section{A}

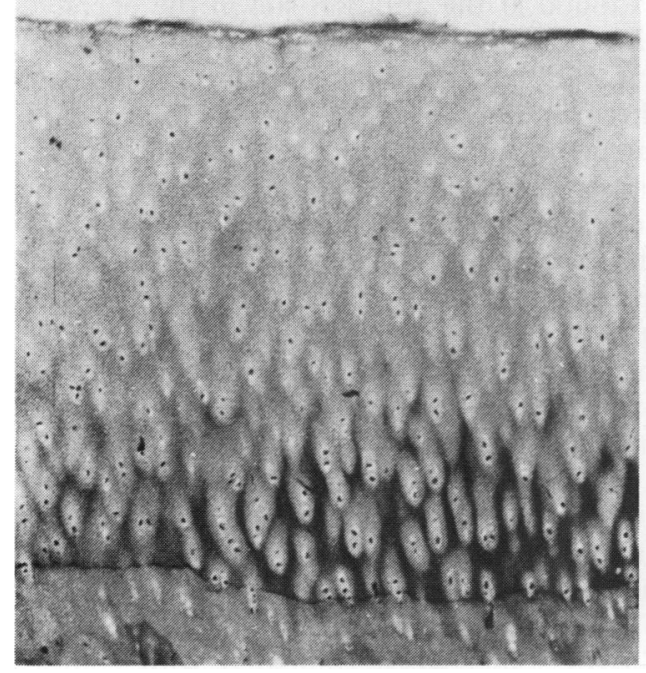

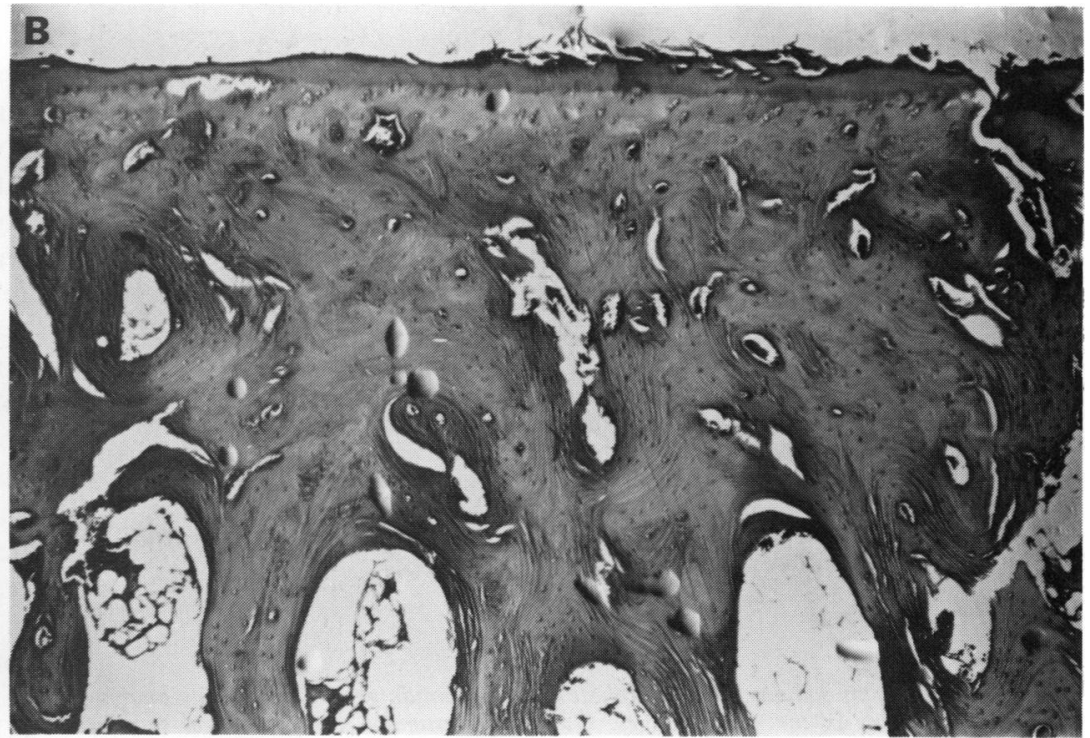

C

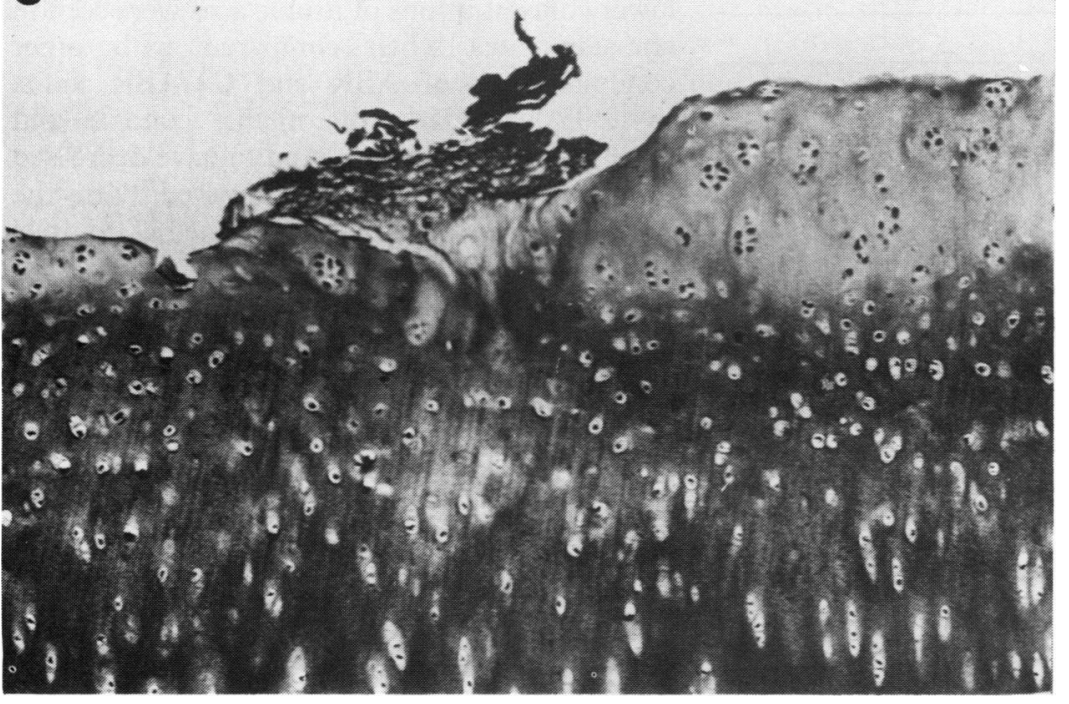

Figure 4 Histological sections stained with safranin $O$ and $a$ fast green counterstain. (A) Non-abraded cartilage from an unoperated control stifle shows a continuous surface, normal cellularity, safranin $O$ staining and intact tidemark. (B) One year after abrasion

chondroplasty the cartilage surface fragments easily, there is reduced cellularity in thin areas of repair tissue, safranin $O$ staining is lost, and the tidemark is redundant and invaded by capillaries. The edge of the abraded area is to the far right. The repair cartilage has the appearance of fibrocartilage. (C) One year after cruciate transection the upper medial femoral condyle is ulcerated. Adjacent articular cartilage shows cell cloning, surface fragmentation, reduced safranin $O$ staining but remaining tidemark integrity. Further to the left of the field there is an area of denuded bone. 
Table 2 Biochemical changes in canine articular cartilage composition with or without abrasion. All values are mean $(S D)$ except for the transected (CT) group

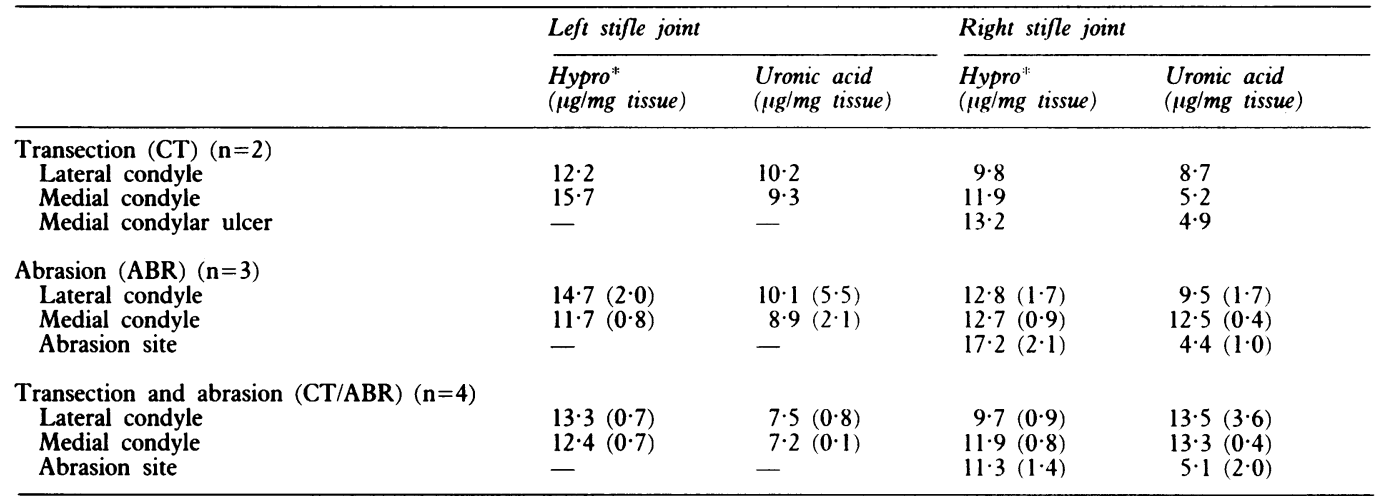

${ }^{*}$ Hypro= hydroxyproline.

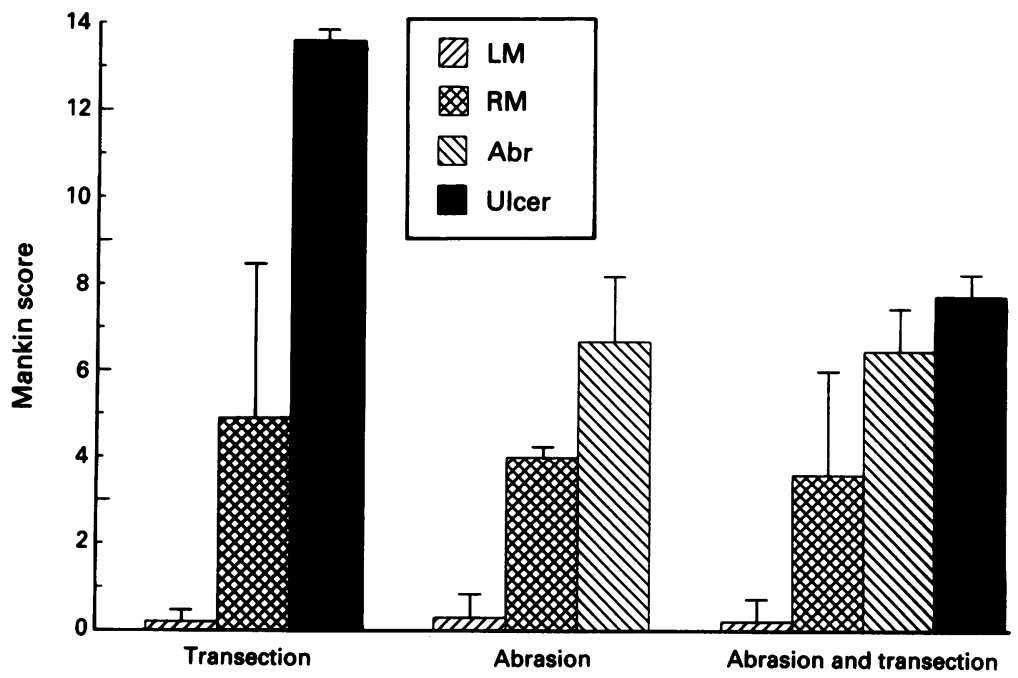

Figure 5 Comparative results of histological histochemical grading (Mankin score; mean (SEM)) of canine articular cartilage one year after anterior cruciate transection, abrasion chondroplasty, or abrasion chondroplasty following anterior cruciate transection. $L M=$ nonoperated left medial condyle used as a control; $R M=$ non-ulcerated cartilage from the operated right medial condyle; $A b r=$ area of repair cartilage over the abrasion chondroplasty sites; Ulcer = cartilage from the upper condylar ulcer appearing after anterior cruciate transection. Readings by Mankin score (see under 'Methods').

Table 3 Summary of sodium dodecylsulphate polyacrylamide gel electrophoresis analyses of cartilage collagens in the repair tissues. Qualitative estimates of relative gel staining intensity for each collagen type. $0=$ not detected on gel; $X X X X$ to $X=$ strong to weak presence (type I collagen assessed by intensity of the $\mathrm{x}_{2}$ (I) chain)

\begin{tabular}{|c|c|c|c|c|c|c|}
\hline & \multicolumn{6}{|c|}{ Left stifle joint (control) } \\
\hline & $I$ & II & $v$ & $V I$ & $I X$ & $X I$ \\
\hline $\begin{array}{l}\text { Abrasion (ABR) }(n=2) \\
\text { Lateral condyle } \\
\text { Medial condyle }\end{array}$ & $\begin{array}{l}0 \\
0\end{array}$ & $\underset{\text { XXXX }}{\operatorname{XxXX}}$ & $\begin{array}{l}0 \\
0\end{array}$ & $\begin{array}{l}0 \\
0\end{array}$ & $\begin{array}{l}0 \\
0\end{array}$ & $\begin{array}{l}\mathbf{X} \\
\mathbf{X}\end{array}$ \\
\hline \multirow[t]{3}{*}{$\begin{array}{l}\text { Transection and abrasion } \\
\text { (CT/ABR) }(n=2) \\
\text { Lateral condyle } \\
\text { Medial condyle }\end{array}$} & $\begin{array}{l}0 \\
0\end{array}$ & $\underset{\mathrm{Xxxx}}{\operatorname{xxxy}}$ & $\begin{array}{l}0 \\
0\end{array}$ & $\begin{array}{l}0 \\
0\end{array}$ & $\begin{array}{l}0 \\
0\end{array}$ & $\stackrel{x}{\mathbf{x}}$ \\
\hline & \multicolumn{6}{|c|}{ Right stifle joint (operated) } \\
\hline & I & II & $\mathrm{v}$ & VI & IX & XI \\
\hline $\begin{array}{l}\text { Abrasion (ABR) }(n=2) \\
\text { Lateral condyle } \\
\text { Medial condyle } \\
\text { Abrasion }\end{array}$ & $\begin{array}{l}0 \\
0 \\
\mathrm{XXXX}\end{array}$ & $\begin{array}{l}\underset{X X X X}{X X X X} \\
\text { XXXX }\end{array}$ & $\begin{array}{l}0 \\
0 \\
\mathrm{x}\end{array}$ & $\begin{array}{l}0 \\
0 \\
\mathrm{X}\end{array}$ & $\begin{array}{l}0 \\
0 \\
0\end{array}$ & $\begin{array}{l}\mathrm{X} \\
\mathbf{X} \\
\mathrm{X}\end{array}$ \\
\hline $\begin{array}{l}\text { Transaction and abrasion } \\
\text { (CT/ABR) }(n=2) \\
\text { Lateral condyle } \\
\text { Medial condyle } \\
\text { Abrasion }\end{array}$ & $\begin{array}{l}\mathbf{0} \\
\mathbf{0} \\
\mathrm{xx}\end{array}$ & $\begin{array}{l}\underset{\operatorname{XXXX}}{\operatorname{XXXX}} \\
\mathbf{X X X}\end{array}$ & $\begin{array}{l}0 \\
0 \\
0\end{array}$ & $\begin{array}{l}0 \\
0 \\
\mathrm{x}\end{array}$ & $\begin{array}{l}0 \\
\mathrm{X} \\
\mathrm{X}\end{array}$ & $\begin{array}{l}\mathrm{XX} \\
\mathbf{X X} \\
\mathbf{X X}\end{array}$ \\
\hline
\end{tabular}

The Mankin scores (fig 5) for ABR left medial condyles (contralateral controls) were normal in histological appearance and had significantly lower scores than either right medial condyles $(\mathrm{p}<0.001)$ or abraded cartilage sites $(\mathrm{p}<0.001)$. The right medial condyles had lower scores than the abraded sites.

HYDROXYPROLINE AND URONIC ACID CONTENT The highest concentration of hydroxyproline was found at the abrasion site in the operated right knee of $A B R$ animals $(p<0.02$ when compared with medial condylar cartilage of either limb) (table 2). The hydroxyproline content of abraded areas in CT/ABR animals was similar to that in other regions of the ipsilateral medial condyle. Cartilage from all transected joints (CT and CT/ABR groups) appeared to contain less hydroxyproline than found in either the ABR joint or any of the contralateral unoperated control joints. This observation presumably reflects an increased water content due to damage to the collagen network and subsequent uptake of water by the resident proteoglycan population. ${ }^{24}$

The highest concentration of uronic acid was found in the medial condyle of ABR and $C T / A B R$ joints as well as the lateral condyle of CT/ABR joints when compared with contralateral sites $(p<0.05$; table 2$)$. Significantly lower concentrations of uronic acid were seen in abrasion sites when compared with other condylar sites of $\mathrm{ABR}$ and $\mathrm{CT} / \mathrm{ABR}$ joints $(p<0.05)$. Similarly the medial condyle and ulcer of CT joints were found to have decreased uronic acid levels when compared with the lateral condyle or contralateral medial condyle of CT joints $(p<0.001)$.

Cartilage from the two abrasion groups (ABR and $(T / A B R$ ) as well as ulcers due to osteoarthritis contained decreased concentrations of uronic acid. The uronic acid/hydroxyproline ratio was less than 0.5 in abraded sites of $A B R$ and $C T / A B R$ joints in addition to the medial condyle and ulcer of the CT group. The ratio was greater than 1.0 in the lateral and medial condyles of the CT/ABR joints. 
extracts of articular cartilage from stifle joints of four dogs is summarised in table 3. Type I collagen was an important component of the repair tissue formed at the abrasion site. Type II collagen was also evident in the repair tissue from all abrasion sites but was less prominent than type I collagen. Type $\mathrm{V}$ collagen rather than type XI collagen was evident in one of the four animals' repair sites and type VI collagen in two of four abrasion sites. Type IX collagen was detected in the CT/ABR medial condyle and abrasion site but was too low to detect by sodium dodecylsulphate polyacrylamide gel electrophoresis in either location in the ABR joints.

\section{Discussion}

Chondral abrasion, alone or in combination with a destabilised joint, induced partial repair of articular cartilage that was evident at six months and which persisted to one year.

When abrasion chondroplasty was performed on a normal joint the repair tissue was soft, fluffy, thin, and abnormal in gross and microscopic appearance. There were isolated areas of denuded bone. Proteoglycan content was low and collagen content was high by the hydroxyproline assay when compared with control articular cartilage. The repair tissue was fibrocartilage by histological appearance, low uronic acid/hydroxyproline ratio, presence of type I collagen, and reduced amounts of type II collagen. Types V and VI collagens were also noted to be prominent as minor collagen species, confirming the fibrocartilaginous nature of the repair tissue.

To simulate a common clinical setting the joint was destabilised as described by Pond and Nuki. ${ }^{22}$ Earlier studies show that cartilage lesions begin to develop as early as two weeks in this model as evidenced by a decrease in proteoglycan aggregation and an increase in metalloproteinase content ${ }^{24}$ (Dean, Muniz, and Howell, unpublished observations). This study did not examine the results of abrasion chondroplasty on more advanced arthritic lesions. At two weeks abrasion chondroplasty was performed to a weightbearing area of the anteroinferior medial surface of the medial condyle. At six months follow up the abraded area had a soft, fluffy cartilage. The latter was continuous with an ulcer that extended anteriorly and proximally in a linear fashion along the medial condyle. This ulcer persisted and was extensive at one year. A second ulcer was present on the proximal anterior medial condyle. This latter ulcer had the same appearance and chemistry as that observed in the knee destabilised by cruciate transaction but without abrasion. ${ }^{24}$ Gross and microscopic anatomy of cartilage from the abraded area was abnormal. The proteoglycan content was reduced as judged by loss of safranin $O$ staining and decreased uronic acid content. Fibrocartilage was suggested by the histological appearance, low uronic acid/hydroxyproline ratio, and presence of type I collagen in one of two specimens. Cartilage distant from these sites was also histologically abnormal by Mankin scoring.
Cartilage from abraded sites of normal and destabilised joints were similar. In contrast to abrasion alone, however, the abrasion sites of the destabilised osteoarthritic joints did not show increased hydroxyproline content. Also unlike abrasion of a stable joint, cartilage from the destabilised osteoarthritic joint was more characteristic of a cartilage phenotype as it contained types II, XI, and IX collagen.

In a similar study by Richmond et al (paper presented at annual meeting of the Orthopaedic Research Society; 1985; Las Vegas, NE) an unstable left stifle was created by anterior cruciate transection and medial meniscectomy with curette of the proximal femoral trochlea. Abrasion chondroplasty was performed on the curetted area four months after cruciate transection. After an additional one to four months the defect was found to be filled with a homogeneous white cartilage of various thicknesses that blended into the surrounding tissue. Part of the matrix stained with safranin $\mathrm{O}$ and the other part was fibrocartilage. These workers felt that preservation of the tidemark might lead to a more normal repair cartilage. Similarly our cartilage was abraded through the tidemark producing a fibrocartilage repair tissue. Bleeding would only occur if the tidemark was violated. Studies in which the tidemark was not penetrated did not yield repair of cartilage defects. ${ }^{13}$

In another study Convery et al created different size defects in horse articular cartilage that exposed cancellous bone. ${ }^{34}$ Small defects ( $3 \mathrm{~mm}$ ) on non-weight bearing surfaces appeared to heal. Similar to our results defects of 9,15 , or $21 \mathrm{~mm}$ repaired partially with fibrous tissue, fibrocartilage, hypercellular cartilage, and occasionally bone. Their hydroxyproline content was inconsistent. Although repair cartilage hexosamine content was less than unoperated controls, this difference was less severe in their study after nine months. Kim et al have reported that $3 \mathrm{~mm}$ defects healed more rapidly in rabbits undergoing continuous passive motion for two weeks after the creation of the defects. ${ }^{35}$

Abrasion chondroplasty is based on the hypothesis that defective cartilage is not replaced unless the subchondral plate is breached. ${ }^{13}$ Following the creation of a defect in the subchondral plate the area fills in with a fibrous granulation tissue (Koide $\mathrm{S}$ et al, paper presented at annual meeting of the Orthopaedic Research Society; 1979; San Francisco, CA) that is, in some studies, gradually transformed into hyaline cartilage. ${ }^{3}$ The repair tissue has the appearance of fibrocartilage and in the burr hole of the Pridie model maintains its structure for several months. ${ }^{349}$ The initial collagen in the repair tissue was type $I$. By six to eight weeks in a rabbit study type II collagen had become predominant over type I but the hexosamine content remained low. ${ }^{4}$ Type I collagen persisted, however, until at least one year. ${ }^{4}$ In another technique small drill holes through the subchondral plate were observed to result in the formation of hyaline cartilage that eventually spread over the bone and resurfaced the areas where cartilage had been lost by disease or injury. 5610 In the present study cartilage at sites adjacent to the abrasion site contained 
increased levels of uronic acid and showed abnormal histology, presumably reflecting metabolic changes in the existing cartilage.

In other studies repair cartilage has been found to be fibrous or hyaline and not able to withstand wear. ${ }^{182036}$ Inadequate repair was believed to be related to penetration into the cancellous bone ${ }^{17}$ during the abrasion procedure. Penetration into the cancellous bone was scrupulously avoided in our animals.

In summary the results of this study show that the quality of the repair cartilage following chondral abrasion varies considerably but was never fully hyaline by biochemical or morphological criteria. The indication that abrasion of cartilage in the destablised joint tended to result in a collagen phenotype more characteristic of cartilage provides hope that under special conditions chondral abrasion will prove of value for the osteoarthritic joint in humans.

We acknowledge the technical assistance of Ofelia Muniz, Sara Morales, Vicky Grayson, Alberto Ballesteros and Agueda Agunde\%, and the secretarial assistance of Michele Chin. This Agunde\%, and the secretarial assistance of Michele Chin. This
work was supported by the Veterans Administration and NIH work was supported by the Veter.
grants AR-08662 and AR 36794.

1 Calandruccio R A, Gilmer W S Jr. Proliferation, regeneration, and repair of articular cartilage of immature animals. f Bone foint Surg [Am] 1962; 44: 431-55.

2 Campbell C J. The healing of cartilage defects. Clin Orthop 1969; 64: 45-63.

3 DePalma A F, McKeever C D, Subin D K. Process of repair of articular cartilage demonstrated by histology and autoradiography with tritiated thymidine. Clin Orthop 1966; 48: $229-42$.

4 Furukawa T, Eyre D R, Koide S, Glimcher M J. Biochemical studies on repair cartilage resurfacing experimental defects in the rabbit knee. F Bone foint Surg [Am] 1980; 62: 79-89.

5 Insall J. The Pridie debridement operation of osteoarthritis of the knee. Clin.Orthop 1974; 101: 61-7.

6 Insall J N. Intraarticular surgery for degenerative arthritis of the knee: a report of the work of the late $\mathrm{K}$. H. Pridie. f Bone foint Surg [Br] 1967; 49: 211-28.

7 Key J A. Experimental arthritis: the changes in joints produced by creating defects in the articular cartilage. f Bone foint Surg 1931; 13: 725-39.

8 Meachim G, Roberts C. Repair of the joint surface from subarticular tissue on the rabbit knee. 7 Anat 1971; 109: 317-27.

9 Mitchell N, Shepard N. The resurfacing of adult rabbit articular cartilage by multiple perforations through the subchondral bone. $\mathcal{F}$ Bone foint Surg [Am] 1976; 58:230-3.

10 Pridie $\mathrm{K} \mathrm{H}$. A method of resurfacing osteoarthritic knee joint. In: Proceedings of the British Orthopaedic Association. J Bone foint Surg [Br] 1959; 41: 618-9.

11 Salter R B, Simmonds D F, Malcolm B W, Rumble E J, MacMichael D. The effects of continuous passive motion on the healing of articular cartilage defects-an experimental investigation in rabbits. In: Proceedings of the Orthopaedic Research Society. F Bone foint Surg [Am] 1975; 57: 570-1.

12 Bert J M, Maschka $\mathrm{K}$. The arthroscopic treatment of unicompartmental gonarthrosis: a five-year follow-up study of abrasion arthroplasty plus arthroscopic debridement and arthroscopic debridement alone. Arthroscopy 1989; 5: 25-32.

13 Burks R T. Arthroscopy and degenerative arthritis of the knee: a review of the literature. Arthroscopy 1990; 6: 43-7.

14 Dandy D J. Abrasion chondroplasty. Arthroscopy 1986; 2: 51-3.

15 Friedman M J, Berasi C C, Fox J M, Del Pizzo W, Snyder S J, Ferkel R D. Preliminary results with abrasion arthroplasty in the osteoarthritic knee. Clin Orthop 1984; 182: 200-5.

16 Johnson L L. Diagnostic and operative arthroscopy of the knee and other joints. St Louis: Mosby, 1981.

17 Johnson L L. Arthroscopic abrasion arthroplasty historical and pathologic perspective: present status. Arthroscopy and pathologic

18 Magnuson P B. Technic of debridement of the knee joint for arthritis. Surg Clin North Am 1946; 26: 249-66.

19 Rand J A. Arthroscopy and articular cartilage defects. Contemporary Orthopaedics 1985; 11: 13-26.

20 Mankin $\mathrm{H} \mathrm{J}$. The reaction of articular cartilage to injury and osteoarthritis. Part I. N Engl f Med 1974; 291: 1285-92.

21 Salter R B, Simmonds D F, Malcolm B W, Rumble E J, MacMichael D, Clements N D. The biological effect of continuous passive motion on the healing of full thickness defects in articular cartilage: an experimental investigation in the rabbit. $\mathcal{F}$ Bone Foint Surg [Am] 1980; 62: 1232-51.

22 Pond M J, Nuki G. Experimentally-induced osteoarthritis in the dog. Ann Rheum Dis 1973; 32: 387-8.

23 Outerbridge R E. The etiology of chondromalacia patellae. f Bone foint Surg [Br] 1961; 43: 752-7.

24 Altman R D, Tenenbaum J, Latta L L, Riskin W, Blanco LN, Howell DS. Biomechanical and biochemical properties of dog cartilage in experimentally induced osteoarthritis. Ann Rheum Dis 1984; 43: 83-90.

25 Mankin H J, Dorfman H, Lippiello L, Zarnis A. Biochemical and metabolic abnormalities in articular cartilage from osteoarthritic human hips. II. Correlation of morphology with biochemical and metabolic data. 7 Bone foint Surg [Am] 1971; 53: 523-37.

26 Oegema T R, Carpenter B J, Thompson R C. Fluorometric determination of DNA in cartilage of various species. F Orthop Res 1984; 1 : 345-51.

27 Bitter T, Muir H M. A modified uronic acid carbazole reaction. Anal Biochem 1962; 4: 330-4.

28 Morales T I, Woessner J F Jr, Howell D S, Marsh J M, LeMaire W J. A microassay for the direct demonstration of collagenolytic activity in graafian follicles of the rat. Biochim Biophys Acta 1978; 524: 428-34

29 Woessner J F Jr. The determination of hydroxyproline in tissue and protein samples containing small proportions of this imino acid. Arch Biochem Biophys 1961; 93: 440-7.

$30 \mathrm{Wu} \mathrm{J}$ J, Eyre D R. Cartilage type IX collagen is cross-linked by hydroxypyridinium residues. Biochem Biophys Res Commun 1984; 123: 1033-9.

31 Laemmli U K. Cleavage of structural proteins during the assembly of the head of bacteriophage T -4 . Nature 1970; 227: $680-5$.

32 Moses L E. Think and explain with statistics. Reading: Addison-Wesley, 1986: 133-64.

33 Kellgren J H, Lawrence J S. Osteo-arthrosis and disk degeneration in an urban population. Ann Rheum Dis 1958; 17: 388-97.

34 Convery F R, Akeson W H, Keown G H. The repair of large osteochondral defects. An experiment study in horses. Clin Orthop 1972; 82: 253-62.

35 Kim H K W, Moran M E, Salter R B. The potential for regeneration of articular cartilage in defects created by chondral shaving and subchondral abrasion. $\mathcal{f}$ Bone foint Surg $[A m]$ 1991; 73: 1301-15.

36 Kempson G E, Muir H, Swanson A, Freeman M A R. Correlations between stiffness and chemical constituents of the human femoral head. Biochim Biophys Acta 1970; 215: $70-7$. 